\title{
The Test-Retest Reliability of Bilateral and Unilateral Force Plate-Derived Parameters of the Countermovement Push-Up in Elite Boxers
}

\author{
Gemma N. Parry, Lee C. Herrington, lan G. Horsley, and lan Gatt
}

\begin{abstract}
Context: Maximal power describes the ability to immediately produce power with the maximal velocity at the point of release, impact, and/or take off-the greater an athlete's ability to produce maximal power, the greater the improvement of athletic performance. In reference to boxing performance, regular consistent production of high muscular power during punching is considered an essential prerequisite. Despite the importance of upper limb power to athletic performance, presently, there is no gold standard test for upper limb force development performance. Objective: To investigate the test-retest reliability of the force plate-derived measures of countermovement push-up in elite boxers. Design: Test-retest design. Setting: High Performance Olympic Training Center. Participants: Eighteen elite Olympic boxers (age $=23$ [3] y; height $=1.68$ [0.39] m; body mass $=70.0$ [17] kg). Intervention: Participants performed 5 repetitions of countermovement push-up trials on FD4000 Forcedeck dual force platforms on 2 separate test occasions 7 days apart. Main Outcome Measures: Peak force, mean force, flight time, rate of force development, impulse, and vertical stiffness of the bilateral and unilateral limbs from the force-time curve. Results: No significant differences between the 2 trial occasions for any of the derived bilateral or unilateral performance measures. Intraclass correlation coefficients indicated moderate to high reliability for performance parameters (intraclass correlation coefficients $=.68-.98)$ and low coefficient of variation $(3 \%-10 \%)$ apart from vertical stiffness (coefficient of variation $=16.5 \%-25 \%)$. Mean force demonstrated the greatest reliability (coefficient of variation $=3 \%)$. In contrast, no significant differences $(P<.001)$ were noted between left and right limbs $(P=.005-.791)$, or between orthodox or southpaw boxing styles $(P=.19-.95)$. Conclusion: Force platform-derived kinetic bilateral and unilateral parameters of countermovement push-up are reliable measures of upper limb power performance in elite-level boxers; results suggest unilateral differences within the bilateral condition are not the norm for an elite boxing cohort.
\end{abstract}

Keywords: upper limb power, Forcedecks, boxing

Upper limb muscular performance has previously been evaluated via medicine ball throws, bench press, and timed push-ups. ${ }^{1-5}$ These methods tend to report power as a measurement of distance thrown rather than as a rate or time quantity, which suggests these tests are more representative of work output, not power produced. These movements also require the incorporation of the whole body, and as such, it is difficult to isolate the specific contribution by the upper limb. With little consensus around what constitutes optimal load and with loading parameters to maximize power output not clearly defined, the mechanisms concerning adaptation following ballistic exercise remains unknown. ${ }^{6,7}$ Plyometric exercises are performed with body mass and are not subjected to methodological study design issues of load selection. Also ballistic in nature, these exercises are distinguished by stretch shortening cycle (SSC) muscle actions. ${ }^{7}$

Athletes who require fast, explosive patterns, upper limb plyometrics, such as the countermovement push-up (CMPU), which optimize the SSC are considered elemental for inducing adaptation, as well as an important component to end stage rehabilitation. ${ }^{1,8,9}$ The plyometric push-up negates the limitations

Parry and Gatt are with GB Boxing, English Institute of Sport, Sheffield, United Kingdom. Parry and Herrington are with the Human Performance Laboratory, Sport, Exercise and Physiotherapy, University of Salford, Greater Manchester, United Kingdom. Herrington and Horsley are with the Physiotherapy Department, English Institute of Sport, Manchester, United Kingdom. Parry (g.parry1@edu.salford.ac. uk) is corresponding author. of the medicine ball throws and bench press throw (BPT), allowing an athlete to explosively displace body mass through a vertical plane ballistic in nature; explosive push-ups do not require the application of a preselected load like BPT.

Similar to a shot-put throw, which when performed in sitting is reported to provide isolated performance of the upper limb, better understanding of upper limb performance is achievable during CMPU due to around only $68 \%$ of body mass being on a force plate. Punching is an immensely explosive, succinct, dynamic action, which occurs during a small period of time ${ }^{10}$; it is proposed that as coaches and clinicians use the CMPU as a training tool to develop muscular power, it could be informative of upper limb muscular power output of a boxer. Rate of force development (RFD), power, and force components play a critical role in plyometric muscular contractions. ${ }^{8}$ Recently, the CMPU or plyometric push up in relation to upper limb performance parameters has been reliably assessed using force-plate- and force-timederived parameters. ${ }^{2-4,11}$

Boxing is a nonsymmetrical sport that requires the development of accuracy, strength, and power. Boxers choose to face their opponent via one of 2 strategies - either via the "southpaw" or "orthodox" stance. During either stance, the boxers will keep their stronger hand at the back to keep the space needed to deliver power punches and the weaker hand at the front for the closer, quicker jabs. ${ }^{10}$

Investigation into lower limb (LL) asymmetry during singleleg jumps indicates that vertical jump performance is better in 
dominant legs compared with nondominant legs. During a CMPU, it would be expected that both arms would add equally to kinematic parameters; however, if side-to-side differences existed, it might be an indicator of physiological adaptation, injury, or deficit in sporting performance by the more utilized side. Following injury, within the clinical environment, injured sides of the body are frequently compared with the noninjured side to assess performance parameters. This is frequently seen with the LL, where the unilateral single-leg hop is compared with the bilateral countermovement jump (CMJ)

Previous studies $2-4,11$ demonstrated good reliability of CMPU-derived parameters; its value as a screening test and evaluator of rehabilitation and condition programs is presently limited. All studies utilized bilateral arm data; no authors presented data on unilateral differences or analyzed differences in CMPU upper limb kinematics in relation to symmetry. If asymmetry is expected when considering dominance, research documenting the relationship between upper limb force platforms-derived parameters and between limbs, asymmetry could provide insight into injury risk, rehabilitation protocols, and areas of power performance development. The aims of this study were two-fold-first to establish if CMPU-derived parameters are reliable for double limb and single limb and second, to establish if unilateral differences occur during bilateral CMPU to inform clinicians how to monitor and assess upper limb performance in relation to athlete monitoring, training program effects, and guide injury rehabilitation. It was hypothesized that CMPU kinetic data will demonstrate good reliability and that asymmetries will exist during CMPU, with data reflecting dominant and nondominant differences in boxing styles.

\section{Methods}

\section{Experimental Approach to the Problem}

Single-group repeated measures design were 5 repetitions of maximal effort CMPU on 2 separate testing sessions 7 days apart. Testing occurred over 21 days, prior to the start of a 6-week powerbased phase, leading towards a major competition.

\section{Participants}

A total of 22 elite male boxers (age $=23$ [3] y; height $=1.68$ [0.39] m; body mass $=70.0$ [17] $\mathrm{kg}$ ) comprising of 2 flyweights, 2 bantamweights, 6 lightweights, 1 welterweight, 2 middleweights, 1 light heavyweight, 2 heavyweight, and 2 super heavyweights participated in this study. Two athletes withdrew due to injury not associated with the upper limb, and 2 withdrew due to attending tournaments. The University of Salford review board approved the investigation, and testing was completed within the spirit of the Declaration of Helsinki, proceeding to test all subjects provided written informed consent.

\section{Procedures}

Five repetitions maximal effort CMPU trials, with 1-minute rest, were completed on 2 separate occasions at the same time of day, in line with previously published protocol. ${ }^{11}$ Each trial was interspersed by a 60-second rest to allow for relocation of the athlete's hands and to eliminate fatigue. ${ }^{8}$ Trials were completed on 2 portable force platforms (FD4000 Forcedeck dual force platforms; VALD Performance, Sydney, Australia) and neuromuscular performance techniques Forcedeck Software (version 1) at a sampling frequency of $1000 \mathrm{~Hz}$. Raw data were analyzed via custom-designed Microsoft Excel Software (Redmond, WA) and filtered of high-frequency noise using a Butterworth low pass filter at $10 \mathrm{~Hz}$. Force plates were zeroed: with weight evenly distributed between both hands, participants adopted a self-selected hand width, shoulder to $90^{\circ}$, torso, legs, and elbows extended, malleolus and feet together. Bodyweight was established from the push-up position. ${ }^{11}$

Following a 3-second countdown, participants immediately lowered their torso rapidly toward the force plate, then immediately pressed vertically as high as possible, aiming for maximal height and trunk elevation, elbows extended hands clearing the force plates, landing back on the force plates with both hands at the same time. Peak force (PF), mean force (MF), RFD, flight time (FT), vertical stiffness (VS), movement time, and impulse were taken from the force-time curve (Figure 1). The methodological recommendations of Maffiuletti et $\mathrm{al}^{8}$ were observed by taking the average of the 3 best efforts for further data analysis to ensure that all variables, notably peak, mean force, and RFD were optimally maximized.

\section{Statistical Analysis}

Reliability of the performance measures between sessions (first aim), paired-sample $t$ tests were performed to deduce any significant changes between trials, intraclass correlation coefficients (ICCs), and within-subject coefficient of variation (CV\%) were calculated with $95 \%$ confidence intervals to determine relationships between test-retest. Boxing style correlational differences were tested by applying the Mann-Whitney $U$ test for side-to-side differences (the second aim). All standard error of the mean and smallest detectable difference were also included to represent and identify the smallest clinically worthwhile change that is statistically significant using SD (pooled) $\times \sqrt{ } 1-$ ICC for standard error of the mean and $1.96 \times \sqrt{ } 2 \times$ standard error of the mean for smallest detectable difference. ${ }^{12}$ Statistical analysis was completed using SPSS (version 23; IBM, Armonk, NY). Data are presented as mean (SD).

\section{Results}

Reliability statistics are presented for each derived parameter in Table 1. Paired sample $t$ tests indicated no significant differences between the 2 trial occasions for all parameters apart from vertical stiffness. The ICCs and within-subject $\mathrm{CV} \%$ calculations indicated substantial to high reliability $(\mathrm{ICC}=.76-.98 ; \mathrm{CV} \%=3 \%-8 \%)$. The smallest detectable difference was large for all parameters $(8.3 \%$

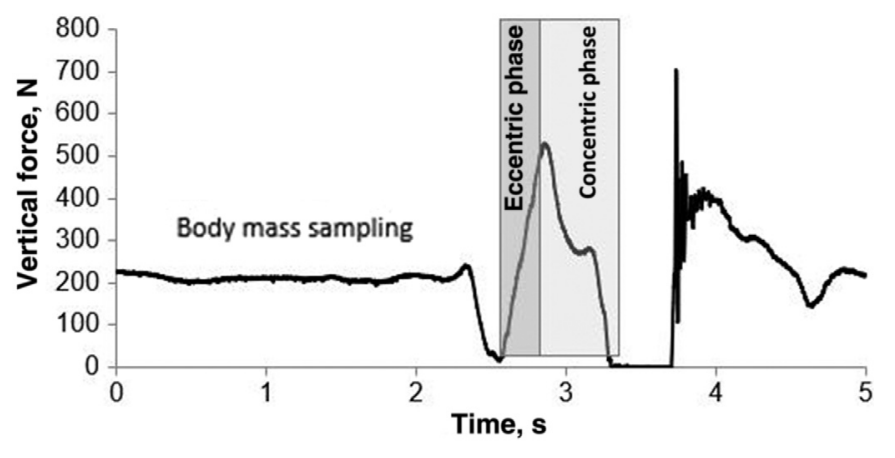

Figure 1 - Example of force-time countermovement push-up curve. 
Table 1 Mean (SD) Values of Reliability for CMPU-Derived Parameters Bilateral and Unilateral Limb $(n=18)$

\begin{tabular}{|c|c|c|c|c|c|c|c|c|}
\hline Derived parameters & Trial 1 & Trial 2 & SEM & CV\% & ICC & $(95 \% \mathrm{Cl})$ & SDD & SDD\% \\
\hline \multicolumn{9}{|l|}{ Bilateral condition } \\
\hline Flight time, ms & $0.7(0.1)$ & $0.7(0.2)$ & 0.1 & 6.9 & .765 & $(.481-.650)$ & 0.2 & 29.5 \\
\hline Peak force, $\mathrm{N}$ & $1012(213)$ & $1009(97)$ & 53.6 & 4 & .929 & $(.821-.902)$ & 148.5 & 14.7 \\
\hline Mean force, $\mathrm{N}$ & $496(113)$ & $495(97)$ & 14.9 & 3 & .978 & $(.892-.943)$ & 41.2 & 8.3 \\
\hline Rate force development, $\mathrm{N} \cdot \mathrm{s}^{-1}$ & $2022(544)$ & $1990(534)$ & 239.1 & 8 & .799 & $(.539-.920)$ & 662.6 & 33 \\
\hline Impulse, $\mathrm{N} \cdot \mathrm{s}$ & $85(34)$ & $83(34)$ & 8.2 & 8 & .94 & $(.849-.977)$ & 22.6 & 26.9 \\
\hline Vertical stiffness, $\mathrm{kN} \cdot \mathrm{m}^{-1}$ & $3.02(1)^{*}$ & $2.82(0.9)^{*}$ & 0.6 & 16.5 & $.563^{*}$ & $(.151-.262)$ & 1.7 & 58.9 \\
\hline \multicolumn{9}{|l|}{ Right limb } \\
\hline Flight time, ms & $0.4(0.1)$ & $0.3(0.1)$ & 0 & 8 & .665 & $(.307-.859)$ & 0.1 & 35.1 \\
\hline Peak force, $\mathrm{N}$ & $510(109)$ & $503(104)$ & 28.7 & 4 & .925 & $(.813-.971)$ & 79.6 & 15.7 \\
\hline Mean force, $\mathrm{N}$ & $252(56)$ & $249(45)$ & 16.9 & 4 & .88 & $(.711-.953)$ & 45.9 & 18.3 \\
\hline Rate force development, $\mathrm{N} \cdot \mathrm{s}^{-1}$ & $1043(296)$ & 994 (297) & 169.3 & 10 & .671 & $(.316-.862)$ & 469.3 & 46.1 \\
\hline Impulse, $\mathrm{N} \cdot \mathrm{s}$ & $42(21)$ & $44(20)$ & 7.8 & 18 & .845 & $(.635-.939)$ & 21.7 & 50.8 \\
\hline Vertical stiffness, $\mathrm{kN} \cdot \mathrm{m}^{-1}$ & $1.49(1)^{*}$ & $1.40(1)^{*}$ & 0.4 & 24 & $.410^{*}$ & $(.064-.731)$ & 1.2 & 81.3 \\
\hline \multicolumn{9}{|l|}{ Left limb } \\
\hline Flight time, ms & $0.4(0.1)$ & $0.4(0.1)$ & 0 & 7 & .783 & $(.511-.913)$ & 0.1 & 31 \\
\hline Peak force, $\mathrm{N}$ & $502(108)$ & $507(103)$ & 28.6 & 4 & .923 & $(.807-.970)$ & 79.4 & 15.7 \\
\hline Mean force, $\mathrm{N}$ & $243(57)$ & $245(55)$ & 7.4 & 3 & .982 & $(.954-.993)$ & 20.5 & 8.4 \\
\hline Rate force development, $\mathrm{N} \cdot \mathrm{s}^{-1}$ & $978(268)$ & $996(265)$ & 114.1 & 9 & .813 & $(.567-.926)$ & 316.4 & 32.1 \\
\hline Impulse, $\mathrm{N} \cdot \mathrm{s}$ & $43(15)$ & $40(18)$ & 7.6 & 17 & .801 & $(.553-.920)$ & 20.9 & 50.6 \\
\hline Vertical stiffness, $\mathrm{kN} \cdot \mathrm{m}^{-1}$ & $1.53(1)^{*}$ & $1.42(1)^{*}$ & 0.3 & 17 & $.688^{*}$ & $(.349-.869)$ & 0.8 & 55.1 \\
\hline
\end{tabular}

Abbreviations: CI, confidence interval; CMPU, countermovement push-up; CV, coefficient of variation; ICC, intraclass correlation coefficient; SDD, smallest detectable difference; SEM, standard error of the mean.

*Significant difference $P \geq .05$.

\section{Table 2 Wilcoxon Signed-Rank Test Results for the Right Versus Left Limb}

\begin{tabular}{lcc}
\hline Derived parameter & $\boldsymbol{P}$ value & $\boldsymbol{r}$ value \\
\hline Flight time, ms & .791 & .10 \\
Peak force, N & .632 & .06 \\
Mean force, N & .005 & .35 \\
Rate of force development, N.s ${ }^{-1}$ & .116 & .20 \\
Impulse, N.s & .314 & .12 \\
Vertical stiffness, $\mathrm{kN} . \mathrm{m}^{-1}$ & .676 & .05 \\
\hline
\end{tabular}

$58.9 \%)$. There were no significant differences between right and left limbs for all derived parameters $(z=0.116-0.791, P=$ .001 ), and differences were trivial (Table 2).

Southpaw style boxers scored higher on FT (mean rank R = 19.13; mean rank $\mathrm{L}=21.31$ ), $\mathrm{RFD}$ (mean rank $\mathrm{R}=20.19$; mean $\operatorname{rank} \mathrm{L}=20.38$ ) and $\mathrm{VS}$ (mean $\operatorname{rank} \mathrm{R}=18.81$; mean $\operatorname{rank} \mathrm{L}=$ 19.56) than orthodox boxing styles (FT: mean rank $\mathrm{R}=18.00$; mean $\operatorname{rank} \mathrm{L}=16.25$; $\mathrm{RFD}$ : mean $\operatorname{rank} \mathrm{R}=17.25$; mean $\operatorname{rank} \mathrm{L}=$ 17.00; VS: mean rank $\mathrm{R}=18.25$; mean rank $\mathrm{L}=17.65)$. Orthodox boxing style demonstrated higher scores of PF (mean rank $\mathrm{R}=$ 19.00; mean rank $\mathrm{L}=19.05$ ), $\mathrm{MF}$ (mean rank $\mathrm{R}=20.55$; mean rank $\mathrm{L}=19.43$ ) and impulse (mean rank $\mathrm{R}=18.60$; mean rank $\mathrm{L}=$ 20.43) than southpaw boxing styles (PF: mean rank $R=17.88$, mean rank $\mathrm{L}=17.81$; $\mathrm{MF}$ : mean $\operatorname{rank} \mathrm{R}=15.94$; mean $\operatorname{rank} \mathrm{L}=$ 17.34; Impulse: mean rank $R=18.38$; mean rank $L=16.09$ ). No statistical differences observed between boxing style groups (Table 3). Effect sizes between parameters and boxing styles were too small for all data (Table 2).

\section{Discussion}

The prime focus of this study was to investigate the test-retest reliability of force-time-derived parameters of the CMPU in both double and single limb in elite boxers. No significant differences $(P>.05)$ were observed between test sessions for all derived parameters apart from vertical stiffness $(P=.01)$. As hypothesized, CMPU reliability was good to excellent (ICC $>.77-.98$ ) for bilateral and unilateral evaluation of right (ICC > .67-.93) and left (ICC $>.79-.98)$ limbs; however, there was no statistical difference between right and left limb or for dominant and nondominant differences in boxing styles. The findings of this study indicate that CMPU bilateral and unilateral force plate-derived parameters are reliable indicators of performance in elite-level boxers.

High reliability $(\mathrm{CV} \%=2.3-11)$ of force-time plyometric push-up is evident within a rugby league population with mean force demonstrating the best reliability $(\mathrm{CV} \%=4.8)$. Moderate to high test-retest reliability (ICC $=.80-.98$ and $.84-.98$ ) respectively for RFD, impulse, and peak average force has also been noted ${ }^{2,3}$ Moreover, 4 variations of plyometric push-ups performed by recreationally active subjects, and active duty marines have also recorded ${ }^{4}$ as having moderate to high test-retest reliability for RFD $(\mathrm{ICC}=.90-.96)$ and peak force (ICC $=.85-.97)$. These authors, ${ }^{2-4}$ along with research on collegiate athletes, ${ }^{11}$ noted that RFD did not demonstrate the same reliability $(\mathrm{CV} \%=11-14.9)$. In contrast, this 
Table 3 Mean (SD) Boxing Styles Comparison of Kinematic-Derived Parameters Between Limbs

\begin{tabular}{|c|c|c|c|c|c|c|c|c|}
\hline \multirow[b]{2}{*}{ Derived parameters } & \multicolumn{4}{|c|}{ Right limb } & \multicolumn{4}{|c|}{ Left limb } \\
\hline & $\begin{array}{l}\text { Orthodox, } \\
\text { mean (SD) }\end{array}$ & $\begin{array}{l}\text { Southpaw, } \\
\text { mean (SD) }\end{array}$ & $\begin{array}{c}P \\
\text { value }\end{array}$ & $\begin{array}{c}r \\
\text { value }\end{array}$ & $\begin{array}{l}\text { Orthodox, } \\
\text { mean (SD) }\end{array}$ & $\begin{array}{l}\text { Southpaw, } \\
\text { mean (SD) }\end{array}$ & $\begin{array}{c}P \\
\text { value } \\
\end{array}$ & $\begin{array}{c}r \\
\text { value } \\
\end{array}$ \\
\hline Flight time, ms & $0.4(0.03)$ & $0.4(0.04)$ & .75 & .053 & $0.3(0.03)$ & $0.4(0.03)$ & .152 & .025 \\
\hline Peak force, $\mathrm{N}$ & $571(21)$ & $499(21)$ & .75 & .053 & $511(15)$ & $496(27)$ & .726 & .058 \\
\hline Mean force, $\mathrm{N}$ & $288(11)$ & $268(4)$ & .191 & .218 & $253(5)$ & $234(7)$ & .556 & .098 \\
\hline $\begin{array}{l}\text { Rate of force develop- } \\
\text { ment, N.s }\end{array}$ & $1100(61)$ & $1073(162)$ & .39 & .145 & $948(73)$ & $1036(105)$ & .34 & .159 \\
\hline Impulse, N.s & $48(5)$ & $41(7)$ & .949 & .158 & $45(7)$ & $36(4)$ & .22 & .036 \\
\hline Vertical stiffness, $\mathrm{kN} \cdot \mathrm{m}^{-1}$ & $1.6(0.3)$ & $1.5(0.3)$ & .873 & .145 & $1.4(0.2)$ & $1.5(0.3)$ & .588 & .098 \\
\hline
\end{tabular}

Note: Significance set at $P<.001$.

study demonstrated better RFD reliability $(\mathrm{CV} \%=$ bilateral $8 \%$, right limb $10 \%$, and left limb 9\%) and less variability than previously reported. This is an important finding, given the importance of RFD to fast, forceful muscle contraction, this study demonstrates that by following a methodological protocol $^{8}$ of sampling at $1000 \mathrm{~Hz}$ and averaging the 3 best trial efforts of 5, RFD can be reliably used to interpret power output performance.

The lack of statistical difference observed between limbs $(P<$ $.001)$ or boxing styles $(P>.05)$ was unanticipated because of punching impact force being a primal performance criterion within elite boxing. ${ }^{10}$ Despite southpaw boxing styles demonstrating higher FT, RFD, and VS and orthodox styles demonstrating higher $\mathrm{PF}, \mathrm{MF}$, and impulse scores, results refute the hypothesis that boxing style dominance difference would associate with right and left limb differences. Rejection of the unilateral difference hypothesis may also be related to equipment used. Force decks are a linked dual force platform device, with software that contains preset performance parameters for common screening tests such as CMJ and drop jump. While a gap observed left between force plates to minimize cross-interference, high-frequency noise remained on all output data. Prior to analysis, data were filtered via a low-pass filter to remove high-frequency noise, as upper limb (UL) forces are less than those observed within the LL due to CMPU utilizing 3 quarters of body weight. ${ }^{4,11}$ It is possible the preset LL parameters and algorithms of the force plates were not sensitive enough to discern the lesser UL forces and motion equations required.

Power output between sides showed no statistical differences for either orthodox or southpaw boxing styles. Differences observed between the mean values of orthodox and southpaw boxers, however, maybe attributed to specific sensorimotor variances required for these contrasting styles. This suggests that used as a bilateral test, both arms would add equally to the kinematic parameters obtained in a CMPU. Despite conditioning practices being bilateral in nature, boxing itself is a nonsymmetrical sport, and differences in both force and power are observed within the literature. ${ }^{10}$ If using the CMPU to monitor training program effects and to guide injury rehabilitation, in relation to physiological adaptation side-to-side differences should not exist within any program aiming to develop equal conditioning. It appears unilateral differences within the bilateral condition are not the norm within this cohort. Any observed unilateral differences in limb performance could potentially indicate injury risk or signs of performance deficit seen through a more reduced score within that side. Practitioners therefore might wish to collect data throughout the year to detect any changes in bilateral performance.
During boxing movements such as jabbing and power punching, the upper limb generates a small portion of the force delivered by the boxer, with the full force occurring due to combined concurrent effort in the upper and lower limbs. ${ }^{13}$ Boxing punches are initiated from the application of force to the ground, with the upper limb segmental extension entirely dependent on the transition of force from the ground through hip and trunk rotation. ${ }^{13}$ While a CMPU is useful for demonstrating upper limb power to further establish asymmetrical differences in boxing styles, future research should analyze additional power measurements such as through a CMJ in addition to CMPU, providing further insight into the generation of explosive power through the punching movement pattern as a whole, and better discern the contribution and relationship of the lower limb to the upper limb power performance within a boxing cohort. There are large interindividual differences in upper limb power due to the range of anthropometrics and characteristics between the different weight categories, which could have impacted the variance of results. Traditionally, power is normalized to $100 \%$ body mass (BM) to reduce bias within results with research ${ }^{10}$ in agreement that athletes of larger strength and size generate greater power outputs. However, unlike the CMJ, which uses $100 \%$ BM, CMPU only involves approximately 3 -quarters $\mathrm{BM}, 4,9,11$ as all subjects' initial mass was averaged on the force plate prior to test commencement, it is argued that all kinematic parameters should be appropriate to each participant's BM.

The results of this study highlight that UL power output in elite-level boxers can be reliably assessed by practitioners using force-plate- and force-time-derived parameters, and unilateral data can be reliably extrapolated from the bilateral condition. When using CMPU to monitor training program affects, no difference between limbs should be noted. This will be useful if completed prior to any injury, as CMPU can be used to better appraise and guide injury rehabilitation until the athlete returns to the improved performance levels. When sampling at $1000 \mathrm{~Hz}$ and averaging 3 best trial efforts of 5, this study's methodology has shown that RFD can be used to reliably interpret UL power output. Future research design should consider similar methodologies to refine the interpretation of RFD within research and practical settings, until then RFD results should continue to be interpreted with caution.

\section{References}

1. McGuigan MR, Wright GA, Fleck SJ. Strength training for athletes: does it really help sports performance? Int J Sports Physiol Perform. 2012;7(1):2-5. PubMed ID: 22461461 doi:10.1123/ijspp.7.1.2 
2. Hogarth L, Deakin G, Sinclair W. Are plyometric push-ups a reliable power assessment tool? J Aust Strength Cond. 2013;21:67-69.

3. Hrysomallis C, Kidgell D. Effect of heavy dynamic resistive exercise on acute upper-body power. J Strength Cond Res. 2001;15(4):426430. PubMed ID: 11726252

4. Koch J, Riemann BL, Davies GJ. Ground reaction force patterns in plyometric push-ups. J Strength Cond Res. 2012;26(8):2220-2227. PubMed ID: 21986698 doi:10.1519/JSC.0b013e318239f867

5. Stockbrugger BA, Haennel RG. Validity and reliability of a medicine ball explosive power test. J strength Cond Res. 2001;15(4):431-438. PubMed ID: 11726253

6. Cormie P, McGuigan MR, Newton RU. Developing maximal neuromuscular power-part 1-biological basis of maximal power production. Sport Med. 2011;41(1):17-38. doi:10.2165/11537690-000000000-00000

7. Cormie P., McGuigan M., Newton R. Developing maximal neuromuscular power part 2 - training considerations for improving maximal power production. Sport Med. 2011;41(2):125-146. doi:10. 2165/11538500-000000000-00000

8. Maffiuletti NA, Aagaard P, Blazevich AJ, Folland J, Tillin N, Duchateau J. Rate of force development: physiological and methodological considerations. Eur J Appl Physiol. 2016;116(6):1091-1116. PubMed ID: 26941023 doi:10.1007/s00421-016-3346-6

9. Moore LH, Tankovich MJ, Riemann BL, Davies GJ. Kinematic analysis of four plyometric push-up variations. Med Sci Sports Exerc. 2011;43(suppl 1):832-843. doi:10.1249/01.MSS.0000402317. 48479.a7

10. Chaabène $H$, Tabben $M$, Mkaouer $B$, et al. Amateur boxing: physical and physiological attributes. Sport Med. 2015;45(3):337-352. doi:10. 1007/s40279-014-0274-7

11. Parry GN, Herrington LC, Horsley IG. The test-retest reliability of force plate-derived parameters of the countermovement push-up as a power assessment tool. J Sport Rehabil. 2019:1-3. doi:10.1123/jsr. 2018-0419

12. Munro A, Herrington L, Carolan M. Reliability of 2-dimensional video assessment of frontal-plane dynamic knee valgus during common athletic screening tasks. J Sport Rehabil. 2012;21(1):7-11. PubMed ID: 22104115 doi:10.1123/jsr.21.1.7

13. Lindsay RS, Lenetsky SM. The contribution of expert coaches' experiential knowledge in understanding punching performance in boxers. J Emerging Sport Stud. 2020;3:1-15. 